Témoigner Témoigner. Entre histoire et mémoire

Getuigen Revue pluridisciplinaire de la Fondation Auschwitz

127 | 2018

Perpétuation de la violence après 1918

\title{
Moi René Tardi. Souvenirs d'un prisonnier de guerre (Jacques Tardi)
}

Ik René Tardi. Herinneringen aan een krijgsgevangenschap (Jacques Tardi)

\section{Brecht Capiau}

Traducteur : Ludovic Pierard

\section{(2) OpenEdition \\ Journals}

\section{Édition électronique}

URL : https://journals.openedition.org/temoigner/7761

DOI : 10.4000/temoigner.7761

ISSN : 2506-6390

Éditeur :

Éditions du Centre d'études et de documentation Mémoire d'Auschwitz, Éditions Kimé

Édition imprimée

Date de publication : 1 octobre 2018

Pagination : 27-31

ISBN : 978-2-930953-076

ISSN : 2031-4183

\section{Référence électronique}

Brecht Capiau, « Moi René Tardi. Souvenirs d'un prisonnier de guerre (Jacques Tardi) », Témoigner. Entre histoire et mémoire [En ligne], 127 | 2018, mis en ligne le 10 février 2022, consulté le 14 février 2022.

URL : http://journals.openedition.org/temoigner/7761 ; DOI : https://doi.org/10.4000/temoigner.7761 


\section{MOI RENÉ TARDI \\ SOUVENIRS D'UN PRISONNIER \\ DE GUERRE (JACQUES TARDI)}

LIVRE En 2012, le dessinateur de bande dessinée français Jacques Tardi publiait Moi René Tardi, prisonnier de guerre au Stalag IIB et sa suite, intitulée Mon retour en France. Cette histoire en deux tomes raconte les tribulations pendant la Seconde Guerre mondiale de René Tardi, le père de Jacques, d'abord membre de l'équipage d'un tank, puis prisonnier de guerre numéro 16402 du Stalag II B d'Hammerstein, en Poméranie orientale. Ce diptyque est probablement l'œuvre la plus thérapeutique de Tardi, un artiste obsédé par les conflits mondiaux et le rôle joué par l'homme ordinaire.

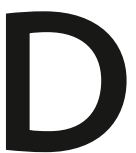
ans ce diptyque, Jacques Tardi se base bien entendu sur les souvenirs de son père, à qui il avait conseillé, en 1980, de mettre par écrit les souffrances qu'il avait endurées pendant la guerre. Mais il fouille également sa propre mémoire et s'insinue lui-même dans la bande dessinée sous les traits d'un enfant en culottes courtes. Ce personnage permet au dessinateur d'entamer un dialogue imaginaire avec René Tardi et de discuter de sujets qui n'avaient jamais été abordés du vivant du père. Souvent, ce dialogue dégénère en dispute, car ces deux têtes de mule tentent d'expliquer la Seconde Guerre mondiale de leur propre point de vue. L'enfant accompagne néanmoins son père, depuis les premiers combats en mai 1940 jusquaux souvenirs de sa femme Henriette à la gare de Valence, le 23 mai 1945, en passant par sa détention dans un stalag. Au fil de son odyssée, René Tardi commente abondamment les événements qui ont conduit à son incarcération et raconte la vie à l'intérieur du Stalag IIB, avec tout le sarcasme qu'on peut en attendre.
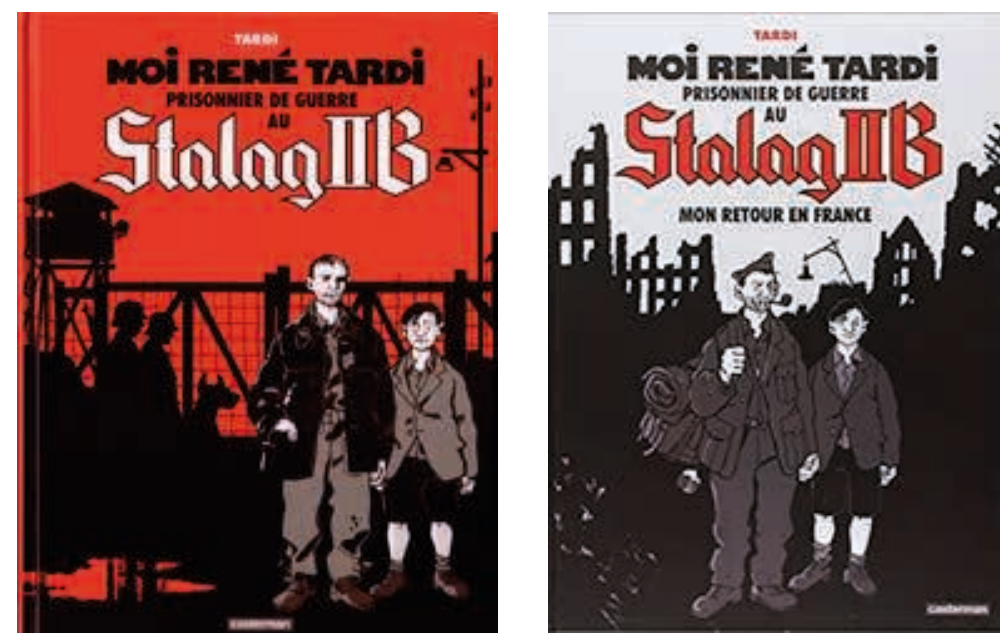

Ce qui frappe immédiatement dans cette bande dessinée, c'est que Jacques Tardi explique souvent à son père des choses qu'il ne peut connaître à ce stade de la narration, mais qui posent un cadre historique plus large. Il appliquera d'ailleurs, fort logiquement, ce procédé rétrospectif dans tout le diptyque. Le choix de l'auteur ne nuit pas aux aspects biographiques de l'histoire, bien au contraire : il augmente l'authenticité du récit de René, car les ajouts historiques alimentent et renforcent le vécu personnel. 

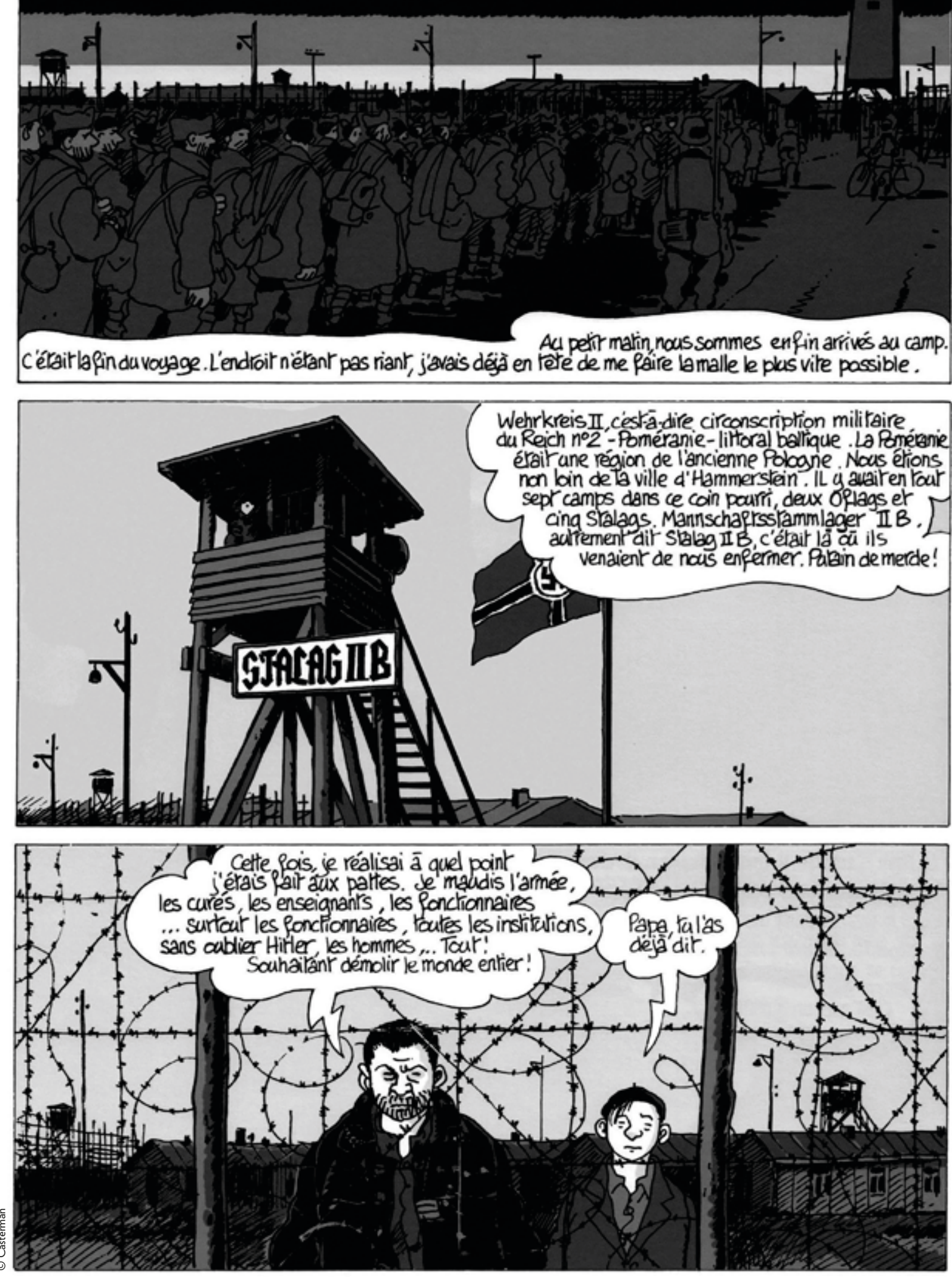

Si le dessinateur s'était déjà révélé être un maitre dans l'art d'illustrer l'atrocité des tranchées pendant a Première Guerre mondiale, il fournit une œuvre d'uns dun stalag. Ses planches panoramiques, complétées par les description extrene père, don de leurs est pratiquent identique à celle des bantes dessine pre Guences réatio

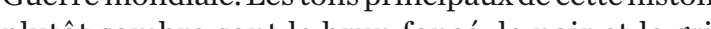
pluto sombre sont le brun fonce, le noir et le gris. Daut 92 du deuxième volet, Mon retour en France. Mas

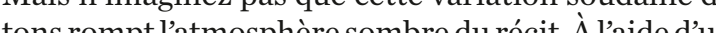
(rouge) sang le dessinater zoome aux pacs 92 stylo (rouge) sang, lessin. pagnons à leurs gardes allemands Les plus hais sont pagnons à lenrs gardes allenands. Les plus hails sont

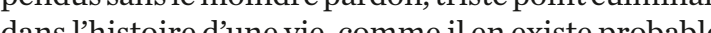
ment beaucoup.

DE VOLONTAIRE À PRISONNIER DE GUERRE

L'histoire du père commence en 1935, lorsqu'il rejoint l'armée en tant que volontaire. Avec René en rejoint larmee en tant que volontaire. Avec Rene en vision quavait le cor den andem tran ision quavait le comm vrir une Frans ses pas, nous nous étonnons de découmeilleure du monde, alors que les ance comme sont formées dans de vieilles carcasses de tacrues

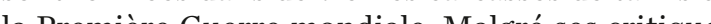
sue l'ét des forces faccises à l'aube de la Seconde Guere mondiale, c'est surtout l'empressement de Gué mondiale, cest surtout l'enpressement de d'en découdre avec les Allemends, il était certience deur flanquer une raclée avec son petit Hotchis le ! I 22 mai 1940, à peine douze jous après l’ . Le 22 mai 19u, à peine donze jours apres linvasion de la Belgique, des Pays-Bas et du Luxembourg enét Turés par les Alle à à près de Péronne, duns la Somme. Le long ép pres de Peronne, dans la Sonnme. Le long et épuisant voyage jusqu'au camp de prisonniers Stalag IIB pe
Bien que Jacques Tardi ait opté dans son dip列 bulations de son père, il soutorise une entorse à vérité au dnoment où René arive dans le camp de tî Delte XII D, à Trêves, ou ilcro bienvent la to de Jean Grange, le futur bearpère de Jacues. Cette de Jean Grange, le futur beau-père de Jacques. Cette certan un jour. En effet, DUlag XII D de TTeves était un Durchounglager biencon Durchyanglager bien conn, c'est-à-dire un lieu de rassemblement pour tous les prisonniers de guerre rés avat d'être trandés Lefaitqué

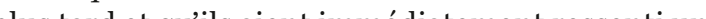
plus tard et quils aient inméd pend pi propule cette histoire, déja intense, au so

Un autre élément frappant est que Jacques Tard dépeint son père tel qu'il était, sans l'idéaliser. Avan la guer il jure tê râle sur tout, et ce n'est pas mient la guere, il jure et rale sur tout, et ce n'est pas nieux pendan de sympathe aù du lecteri, ce qui le rendout de sympathie auprès du lecteur, ce qui le ren , ent nous nens aucune predisposition a lihèroisme et cara a l'pres pas à une gerre qui net notre conste à lépreuve. Mas lorsque ça arive, on leur vie tele quelle ́tait gensanant. Dans un ausi, il est question de train-train, mêne sice n'est plinage que sen fit le grand public. On pense pas limage que s'en fait le grand public. On pense en tels que Stalag 17 (Billy Wilder, 1953) ou $L a$ Grane, tera (Johy 17 (Billy Wilder, 1953) on La Grande toù (Joh Stron ent

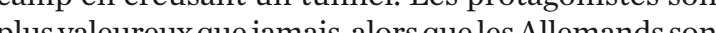
dép al cureuxqu jus alour vepeints sousleur plus nauvais jour. Lesconditions de dée diffiles dans un stalag moyen sont à peine aborde l'évas toujours subore C'est don à to plan l'hoution de plus réal tine faisaient aussi partie de l'existence quotidienne. ... 

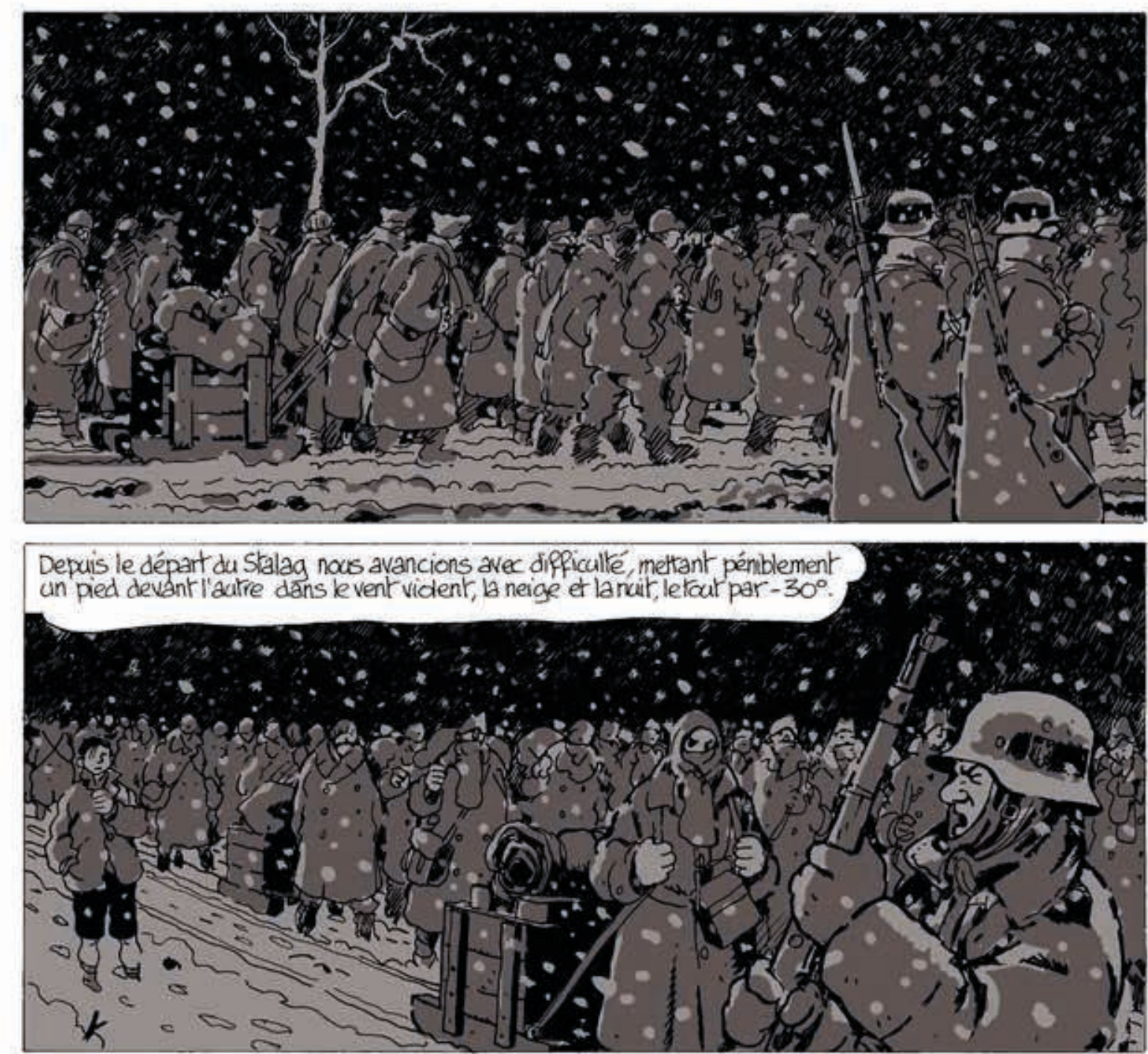

$\because$ DE PRISONNIER DE GUERRE À HOMME LIBRE

Bien que le style narratif et descriptif de Jacques Tardi soit une bénédiction pour le récit il est malheureusement aussiune malédiction pour les phylactères. Certaines planches sont à moitié recouvertes par une bulle, ce qui donne l'impression de lire un roman illus tré plutôt qu'une bande dessinée. Mais Jacques Tardi jouit d'un tel crétit auprès de ses lecteucs Tarvient à parvient à s'en tirer. Du noins dans le premier volet, Mon ren prance est un vai cau Stalag IIB, Car Mon retour en Fresce phylacteres sonten plus surmontés de descriptions de du Stalug, cevif fine fortén du Stalag, ce quf média íné qu suit d'u Une bande dessincée est un mén plus particuli à la fin de tion d'un troisì A lan de cet albú Ty, il st fait menAllemagne, mais qui n'est pas encore paru.

Moi René Tardi, prisonnier de guerre au Stalag IIB et Mon retour en France forment ensemble le récit très personnel du père du dessinateur de bande de dessinée français Jacques Tardi sur sa détention dans le Stalag IIB. Dans la longue préface de ce diptyque, Dominique Grange, l'épouse de Jacques Taty Dominique Grange, I tépouse de Jacques 'ardi, nous Grange par sa famille après la guerre. Il fut confronté Grange par sa famille après la guerre. If fut confronté aux moquen as amères et à lincompréhension, car le grand-pere naternel avait surect aux tranchées de la Prenière Guerre mondiale et navait pas la moindre indulgent que bien des annes plus tard que Jean, qui s'etait du soutien du rès respé du soutien du très respecté René Tari. Ce dernier avait échappe à ce genre de retour et put, contrai-

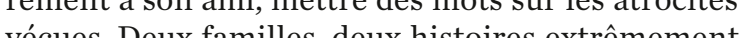
vécues. Deux familles, deux histoires extrêmement captivantes. Neannoins, cellede Jean Grange, malgre le témoignage courageux de sa fille Dominique, se perd de RenéTardipa de René Tardi parmi un club select de bandes dessinees Brecht Capiau
Traduit du néerlandais par Ludovic Pierard

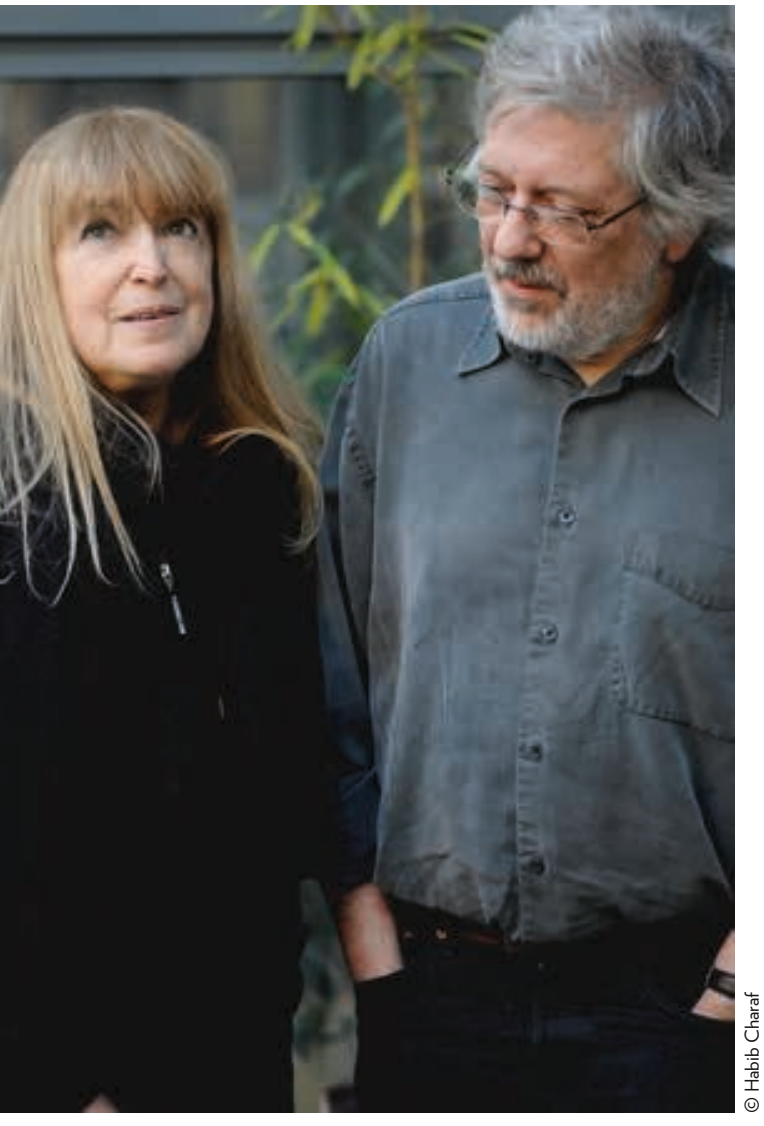

Dominique Grange et Jacques Tard

$\rightarrow$ Bibliographie

$\triangle{ }_{\text {Jacques Tardi, Moi René Tardi, prisonnier de guerre au }}$

$\diamond$ Jacques Tardi, Moi René Tardi, prisonnier de guerre au
Stalag llB Mon retour en France, Bruxelles, Casterman, 2015. 